CRYSTALLOGRAPHIC COMMUNICATIONS

ISSN 2056-9890

Received 25 March 2015

Accepted 13 April 2015

Edited by M. Weil, Vienna University of Technology, Austria

Keywords: crystal structure; hydrogen bonding; structural systematics; XPac; salicylic acid derivative

CCDC reference: 1059331

Supporting information: this article has supporting information at journals.iucr.org/e

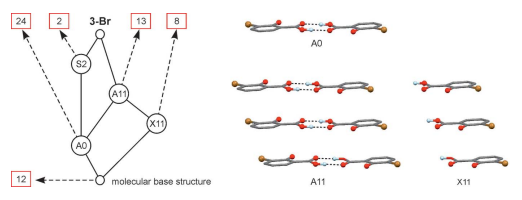

OPEN $\odot$ ACCESS

\section{Crystal structure of 3-bromo-2-hydroxybenzoic acid} Gerhard Laus, ${ }^{a}$ Volker Kahlenberg, ${ }^{b}$ Thomas Gelbrich, ${ }^{a *}$ Sven Nerdinger ${ }^{c}$ and
Herwig Schottenberger ${ }^{a}$

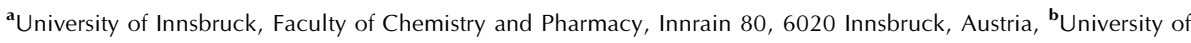
Innsbruck, Institute of Mineralogy and Petrography, Innrain 52, 6020 Innsbruck, Austria, and ' ${ }^{\mathbf{S}}$ andoz GmbH, Biochemiestrasse 10,6250 Kundl, Austria. *Correspondence e-mail: thomas.gelbrich@uibk.ac.at

Mutual carboxyl-carboxyl $\mathrm{O}-\mathrm{H} \cdots \mathrm{O}$ hydrogen bonds link the molecules of the title compound, $\mathrm{C}_{7} \mathrm{H}_{5} \mathrm{BrO}_{3}$, into centrosymmetric dimers which display a central $R_{2}^{2}(8)$ ring motif. In addition, there is an intramolecular hydroxyl-carboxyl O$\mathrm{H}$. . O interaction present. A comparison with the crystal structures of 59 other substituted derivatives of salicylic acid shows that both the centrosymmetric carboxyl-carboxyl $\mathrm{O}-\mathrm{H} \cdots \mathrm{O}$ dimer and the stacking mode of molecules along the short $a$ axis observed in the title structure are frequent packing motifs in this set.

\section{Chemical context}

Substituted derivatives of salicylic acid are widely used in organic synthesis and can be biologically active. Members of this class have served as model compounds for studies of crystal polymorphism (Sarma et al., 2010; Braun et al., 2011), the stability of hydrogen bonds (Bawa et al., 2004; Adam et al., 2010) or for systematic investigations of crystal-packing relationships (Montis \& Hursthouse, 2012). The title compound is used in the synthesis of 7-bromobenzoxazolin-2one (Laus et al., 2011), which is an intermediate in the synthesis of bifeprunox, an experimental drug for the treatment of psychiatric disorders such as schizophrenia (Zwier et al., 2005; Eijgendaal et al., 2006).<smiles>O=C(O)c1cccc(Br)c1O</smiles>

\section{Structural commentary}

The molecule is almost planar (Fig. 1). The plane defined by the non-H atoms of the carboxyl group is slightly twisted by $4.7(4)^{\circ}$ to the mean plane of the phenyl ring. An intramolecular hydrogen bond, $\mathrm{O} 1-\mathrm{H} 1 \cdots \mathrm{O} 3$ (Table 1), connects the hydroxyl group bonded to $\mathrm{C} 2$ with the carboxyl group at C1.

\section{Supramolecular features}

Neighbouring molecules are linked to one another by a twopoint $\mathrm{O} 2-\mathrm{H} 2 \cdots \mathrm{O} 3(-x+2,-y,-z+1)$ connection involving 


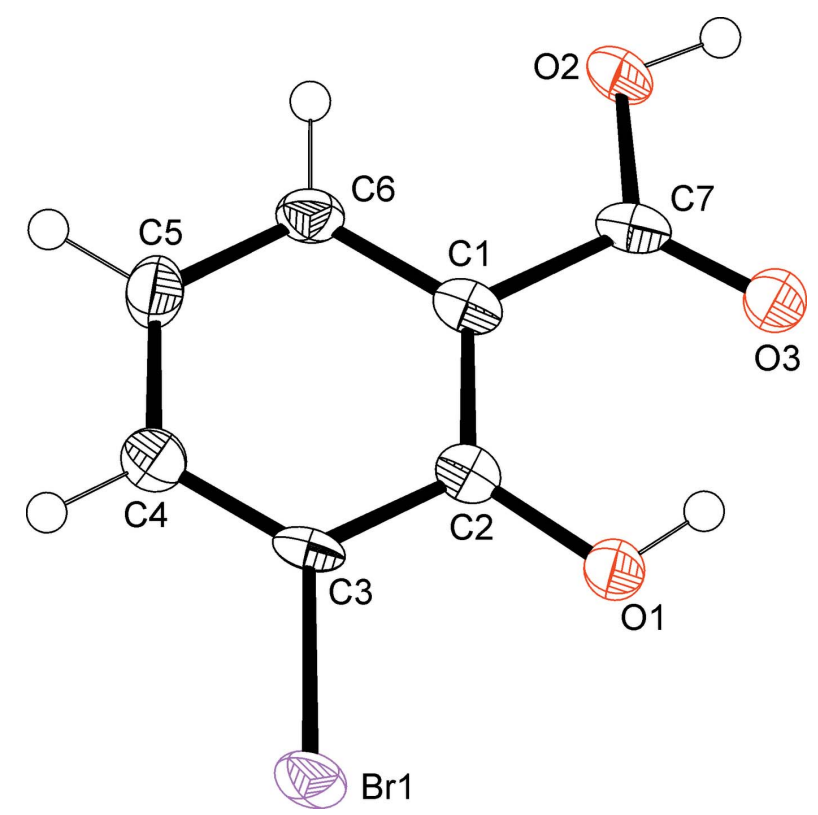

Figure 1

The molecular structure of the title compound, drawn with displacement ellipsoids at the $50 \%$ probability level. $\mathrm{H}$ atoms are drawn as spheres of arbitrary size.

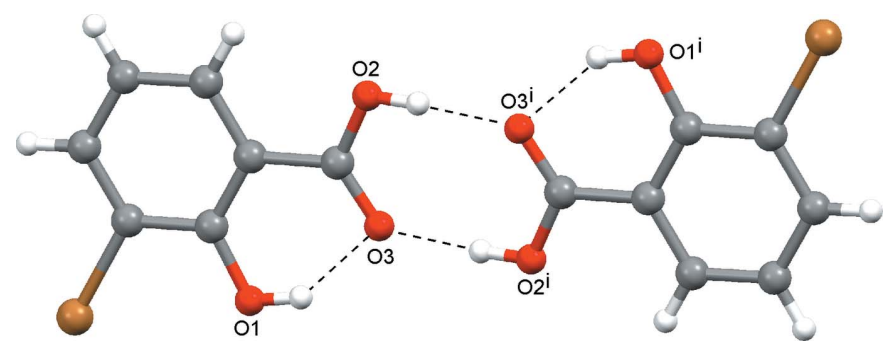

Figure 2

Centrosymmetric dimer with a central $R_{2}^{2}(8)$ ring motif. Dashed lines indicate hydrogen bonds. [Symmetry code: (i) $-x+2,-y,-z+1$.]

a pair of antiparallel interactions between their carboxyl groups (Fig. 2, Table 1). The resulting centrosymmetric dimer is described by the graph set $R_{2}^{2}(8)$ (Etter et al. 1990; Bernstein et al., 1995). In the crystal structure, the dimers, which are essentially planar units, assemble into slightly corrugated sheets which lie parallel to the $(10 \overline{3})$ plane. A sheet of this kind contains a short $\mathrm{C} 4-\mathrm{H} 4 \cdots \mathrm{O} 1\left(-x+\frac{1}{2}, y+\frac{1}{2},-z+\frac{1}{2}\right)$ contact $\left(\mathrm{H} \cdots \mathrm{O}=2.57 \AA, \mathrm{C}-\mathrm{H} \cdots \mathrm{O}=145.7^{\circ}\right)$ (Fig. $\left.3 a\right)$ which involves the hydroxyl group at $\mathrm{C} 2$. The sheets are stacked in a parallel fashion in the $a$-axis direction with an intersheet separation of 3.798 (4) $\AA$ which corresponds to the length of this axis.

Table 1

Hydrogen-bond geometry $\left(\AA{ }^{\circ}\right)$.

\begin{tabular}{lllll}
\hline$D-\mathrm{H} \cdots A$ & $D-\mathrm{H}$ & $\mathrm{H} \cdots A$ & $D \cdots A$ & $D-\mathrm{H} \cdots A$ \\
\hline $\mathrm{O} 1-\mathrm{H} 1 \cdots \mathrm{O} 3$ & $0.85(2)$ & $1.88(4)$ & $2.604(4)$ & $142(5)$ \\
$\mathrm{O} 2-\mathrm{H} 2 \cdots \mathrm{O}^{\mathrm{i}}$ & $0.87(2)$ & $1.80(2)$ & $2.664(4)$ & $172(6)$ \\
\hline
\end{tabular}

Symmetry code: (i) $-x+2,-y,-z+1$.

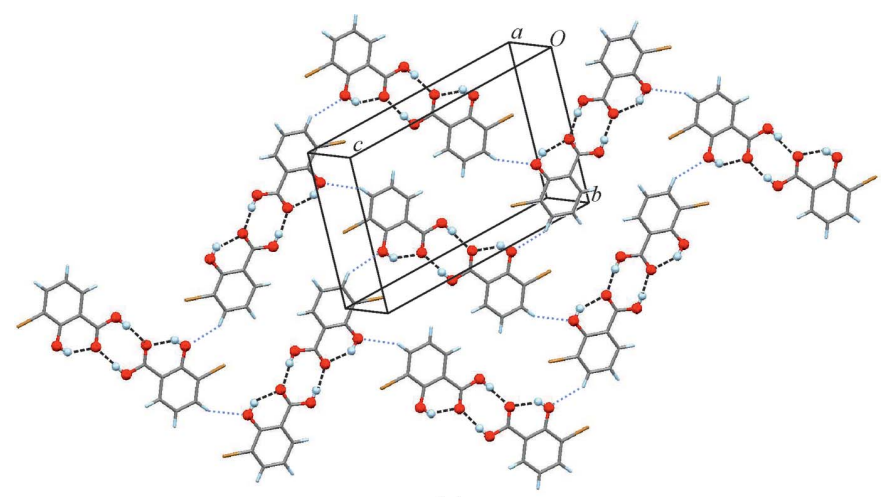

(a)

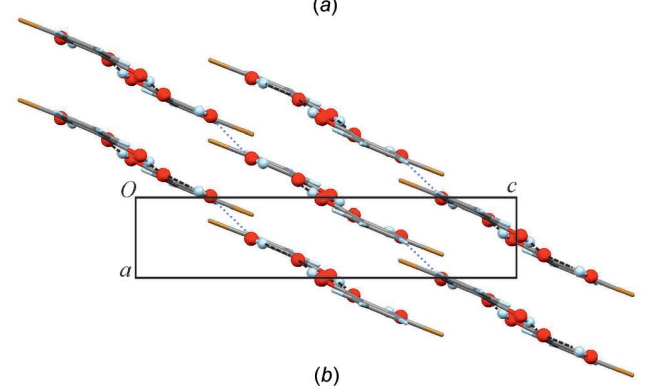

(b)

Figure 3

(a) Molecular packing in the $(10 \overline{3})$ plane. Dashed lines indicate $\mathrm{O}-$ $\mathrm{H} \cdots \mathrm{O}$ hydrogen bonds; $\mathrm{C} 4-\mathrm{H} 4 \cdots \mathrm{O} 1\left(-x+\frac{1}{2}, y+\frac{1}{2},-z+\frac{1}{2}\right)$ contacts are indicated by thin dotted lines. Similar arrangements are present in the crystal structures of $3,5-\mathrm{Br}$ and $3,5-\mathrm{Cl}$ (supramolecular construct S2). (b) Three corrugated sheets stacked in the $a$-axis direction.

\section{Database survey}

A systematic study of packing motifs present in 24 crystal structures of monosubstituted derivatives of salicylic acid has previously been published by Montis \& Hursthouse (2012), who also proposed a nomenclature where a substituent $R^{n}$ at a ring position $n(n=3,4,5$ or 6$)$ is encoded $n-R^{n}$ (Fig. 4; $\left.R^{n} \neq \mathrm{H}\right)$. The title compound of the present study is denoted $3-\mathrm{Br}$ in this system. Our own survey of the Cambridge Structural Database (version 5.25; Groom \& Allen, 2014) revealed 59 unique crystal structures of salicylic acid derivatives, listed in Table S1 of the Supporting information, which are close structural analogues of 3-Br. This set includes several polysubstituted derivatives as well as multiple-component crystals and crystal structures containing potential hydrogen bond<smiles>[R5]c1c([R6])c([R6])c(C(=O)O)c(O)c1[R6]</smiles>

Figure 4

Scheme showing the general composition of substituted derivatives of salicylic acid, the crystal structures of which were compared in this study. 

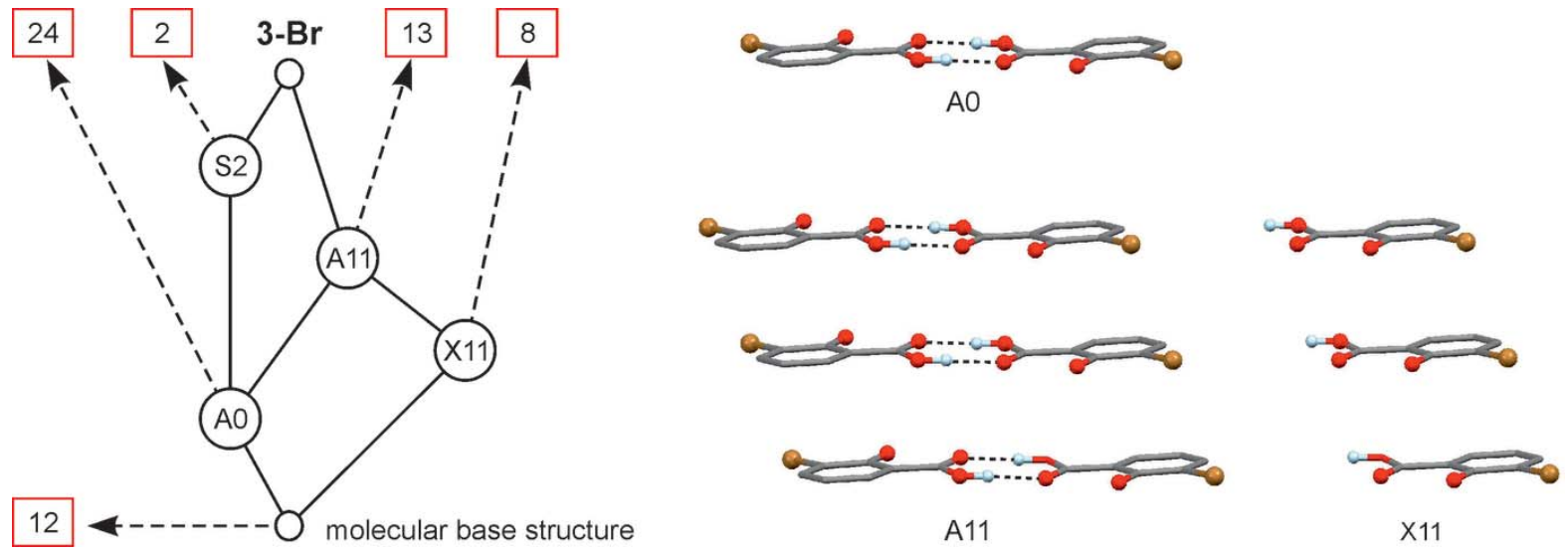

Figure 5

(Left) Tree diagram illustrating the packing relationships between 3-Br and other substituted derivatives of salicylic acid. A number in a box indicates the number of crystal structures for which a given SC (A0, X11, A11 and S2) is the largest common SC with 3-Br. (Right) Instances of the SCs A0, X11, A11 in $3-\mathrm{Br}$.

donor and acceptor sites in addition to those of the 1-hydroxyl and 2-carboxyl groups.

In order to establish the possible existence of geometrically similar substructure units, pairwise XPac comparisons (Gelbrich \& Hursthouse, 2005) were carried out between the crystal structure of $3-\mathrm{Br}$ on one hand and each of the other 59 salicylic acid derivatives on the other. Analogous to the study by Montis \& Hursthouse (2012), the underlying calculations were based on the comparison of intermolecular geometrical parameters generated from the ten non-H atomic positions of the salicylic acid molecular core $\left(\mathrm{C}_{7} \mathrm{O}_{3}\right)$ which is present in all compounds of the set. A quantitative descriptor, the dissimilarity index $x_{10}$ (Gelbrich et al., 2012), was calculated for each common supramolecular construct (SC) identified. In general, a larger $x_{10}$ value indicates a lower degree of similarity and an $x_{10}$ value significantly higher than 10 is consistent with a situation where the fundamental features of a $3-\mathrm{Br}$ substructure unit are also present in a second crystal, albeit with considerable geometrical modifications.

41 structures of the investigated set, including $3-\mathrm{Br}$, contain (carboxyl) $\mathrm{O}-\mathrm{H} \cdots \mathrm{O}_{\text {(carboxyl) }}$ hydrogen-bonded dimers with a central $R_{2}^{2}(8)$ ring motif (Fig. 2). All of the dimers are centrosymmetric, except for that of 3-COOH (Mereiter et al., $2001)$. In the latter structure, the $R_{2}^{2}(8)$ ring motif is intersected by a glide plane and connects the 2-carboxyl group of one molecule with the 3-carboxyl group of the other so that an hydrogen-bonded chain structure is formed. Owing to the rigidity of the aromatic ring and the limited rotational flexibility about the $\mathrm{C} 1-\mathrm{C} 7$ bond, all 40 centrosymmetric dimers adopt approximately the same geometry, and the corresponding $x_{10}$ values are smaller than 12 (Table S1 of the Supporting information). In keeping with the nomenclature introduced by Montis \& Hursthouse (2012), we denote this dimer SC A0.

A one-periodic SC, denoted X11 by Montis \& Hursthouse (2012), describes the stacking of 3-Br molecules along the shortest crystallographic axis [ $a$ axis; Fig. 5(right)]. We have identified another 21 examples of the same stacking mode
(Table S1 of the Supporting Information) and the 13 best matches with $x_{10}>12$ are listed in Table 2. In this latter subset, the length of the stacking vector varies from 3.67 to $3.98 \AA$. Moreover, the eleven structures listed in the top section of Table 2 also contain a centrosymmetric dimer so that their common SC is actually a stack of dimers [denoted A11; Fig. 5(right)].

Other noteworthy packing relationships exist between $3-\mathrm{Br}$ and the structures of 3,5-Br (XISGEM; Liu et al., 2008) and 3,5-Cl (WECXAE; Gao et al., 2005). These are based on the sheet structure which lies parallel to $(10 \overline{3})$ in the $3-\mathrm{Br}$ crystal

Table 2

One-dimensional packing relationships between $3-\mathrm{Br}$ and other derivatives of salicylic acid, based on the stacking of either individual molecules (X11) or dimers (A11) along the short crystallographic axis and identified with XPac.

\begin{tabular}{|c|c|c|c|c|c|}
\hline Compound & $\mathrm{SC}^{a}$ & $x_{10}^{b}$ & $d(\AA)^{c}$ & CSD code & reference \\
\hline $3-\mathrm{Br}$ & A11 & - & 3.80 & - & This work \\
\hline $5-\mathrm{F}$ & A11 & 4.2 & 3.82 & ABENEB & $\begin{array}{l}\text { Choudhury \& Guru Row } \\
\text { (2004) }\end{array}$ \\
\hline $5-\mathrm{COOH}$ & A11 & 4.8 & 3.68 & OJICEP & Cox \& Murphy (2003) \\
\hline $3,4-\mathrm{OH} \cdot 0.25 \mathrm{H}_{2} \mathrm{O}$ & A11 & 5.6 & 3.73 & LAPZUZ & Li et al. (2012) \\
\hline $5-\mathrm{OMe}$ & A11 & 5.6 & 3.98 & VAXZUR & $\begin{array}{l}\text { Montis \& Hursthouse } \\
\text { (2012) }\end{array}$ \\
\hline $5-\mathrm{Cl}$ & A11 & 7.1 & 3.71 & VABVAX01 & $\begin{array}{l}\text { Montis \& Hursthouse } \\
\text { (2012) }\end{array}$ \\
\hline $4-\mathrm{Cl}$ & A11 & 9.2 & 3.72 & VAXYAW & $\begin{array}{l}\text { Montis \& Hursthouse } \\
\text { (2012) }\end{array}$ \\
\hline $5-\mathrm{NO}$ & A11 & 9.5 & 3.67 & NTSALA & Talberg (1977) \\
\hline $4-\mathrm{NH}_{2}$ & A11 & 10.3 & 3.73 & AMSALA02 & $\begin{array}{l}\text { Montis \& Hursthouse } \\
\text { (2012) }\end{array}$ \\
\hline $4-\mathrm{OH}$ & A11 & 10.7 & 3.69 & ZZZEEU04 & Parkin et al. (2007) \\
\hline 4-Me & A11 & 11.5 & 3.87 & VAXYIE & $\begin{array}{l}\text { Montis \& Hursthouse } \\
\text { (2012) }\end{array}$ \\
\hline $5-\mathrm{ACM} \cdot \mathrm{H}_{2} \mathrm{O}$ & X11 & 2.2 & 3.75 & VAXYOK & $\begin{array}{l}\text { Montis \& Hursthouse } \\
\text { (2012) }\end{array}$ \\
\hline $5-\mathrm{CHO}$ & X11 & 5.6 & 3.78 & UJOFI & Lu et al. (2010) \\
\hline $3-\mathrm{CHO} \cdot \mathrm{H}_{2} \mathrm{O}$ & X11 & 11.1 & 3.72 & JOHXEJ & Claude et al. (1991) \\
\hline
\end{tabular}

Notes: $(a)$ the largest supramolecular construct which a crystal has in common with that of 3-Br; (b) XPac dissimilarity index computed from intermolecular geometrical parameters which were calculated using the ten non-H atomic positions of the common salicylic acid molecular fragment; $(c)$ the length of the X11 stacking vector. 
Table 3

Experimental details.

\begin{tabular}{|c|c|}
\hline \multicolumn{2}{|l|}{ Crystal data } \\
\hline Chemical formula & $\mathrm{C}_{7} \mathrm{H}_{5} \mathrm{BrO}_{3}$ \\
\hline$M_{\mathrm{r}}$ & 217.02 \\
\hline Crystal system, space group & Monoclinic, $P 2_{1} / n$ \\
\hline Temperature $(\mathrm{K})$ & 173 \\
\hline$a, b, c(\AA)$ & $\begin{array}{l}3.7978(4), 10.5567(6), \\
\quad 18.0366(10)\end{array}$ \\
\hline$\beta\left(^{\circ}\right)$ & $90.208(7)$ \\
\hline$V\left(\AA^{6}\right)$ & $723.12(10)$ \\
\hline$Z$ & 4 \\
\hline Radiation type & Мо $K \alpha$ \\
\hline$\mu\left(\mathrm{mm}^{-1}\right)$ & 5.63 \\
\hline Crystal size $(\mathrm{mm})$ & $0.32 \times 0.16 \times 0.08$ \\
\hline \multicolumn{2}{|l|}{ Data collection } \\
\hline Diffractometer & $\begin{array}{l}\text { Agilent Xcalibur (Ruby, Gemini } \\
\text { ultra) }\end{array}$ \\
\hline Absorption correction & $\begin{array}{l}\text { Multi-scan (CrysAlis PRO; } \\
\quad \text { Agilent, 2012) }\end{array}$ \\
\hline$T_{\min }, T_{\max }$ & $0.094,1$ \\
\hline $\begin{array}{l}\text { No. of measured, independent and } \\
\text { observed }[I>2 \sigma(I)] \text { reflections }\end{array}$ & $4627,1594,1309$ \\
\hline & 0.040 \\
\hline$(\sin \theta / \lambda)_{\max }\left(\AA^{-1}\right)$ & 0.684 \\
\hline \multicolumn{2}{|l|}{ Refinement } \\
\hline$R\left[F^{2}>2 \sigma\left(F^{2}\right)\right], w R\left(F^{2}\right), S$ & $0.040,0.100,1.08$ \\
\hline No. of reflections & 1594 \\
\hline No. of parameters & 108 \\
\hline No. of restraints & 2 \\
\hline $\mathrm{H}$-atom treatment & $\begin{array}{l}\mathrm{H} \text { atoms treated by a mixture of } \\
\text { independent and constrained } \\
\text { refinement }\end{array}$ \\
\hline$\Delta \rho_{\max }, \Delta \rho_{\min }\left(\mathrm{e} \AA^{-3}\right)$ & $1.19,-0.79$ \\
\hline
\end{tabular}

Computer programs: CrysAlis PRO (Agilent, 2012), SUPERFLIP (Palatinus \& Chapuis, 2007), SHELXL2014 (Sheldrick, 2015), ORTEP-3 for Windows and WinGX (Farrugia, 2012), Mercury (Macrae et al., 2006), XP (Sheldrick, 2008) and publCIF (Westrip, 2010).

and is depicted in Fig. $3 a$. The corresponding $x_{10}$ values of 11.8 and 12.4 for this 2-periodic SC [denoted S2 in Fig. 5(left)] indicate a relaxed form of geometrical similarity, which is consistent with the accommodation of additional halogen substituents in the planes of 3,5-Cl and 3,5-Br. Moreover, the short $\mathrm{C} 4-\mathrm{H} 4 \cdots \mathrm{O} 1$ contact found in $3-\mathrm{Br}$ (see above) is replaced by other close contacts in the $\mathrm{S} 2$ instances of $3,5-\mathrm{Br}$ and 3,5-Cl. Table S2 of the Supporting information contains the corresponding crystallographic parameters associated with SC S2. A graphical overview of the packing relationships involving $3-\mathrm{Br}$ and their interdependencies is given in Fig. 5(left).

\section{Synthesis and crystallization}

The title compound was prepared from 5-sulfosalicylic acid by bromination, followed by desulfonation in hot phosphoric acid and, finally, purification by steam distillation, as described by Meldrum \& Shah (1923). Single crystals were obtained by recrystallisation from hot water.

${ }^{1} \mathrm{H}$ NMR (DMSO- $\left.d_{6}, 300 \mathrm{MHz}\right): 6.87(t, J=7.9 \mathrm{~Hz}, 1 \mathrm{H}), 5.3$ $(b r, 1 \mathrm{H}), 7.80(d, J=7.9 \mathrm{~Hz}, 2 \mathrm{H}), 11.5(b r, 1 \mathrm{H})$ p.p.m. ${ }^{13} \mathrm{C} \mathrm{NMR}$ (DMSO- $\left.d_{6}, 75 \mathrm{MHz}\right): 110.1,114.3,120.2(\mathrm{CH}), 129.7(\mathrm{CH})$, $138.5(\mathrm{CH}), 157.6,171.6$ p.p.m. ${ }^{1} \mathrm{H}$ NMR $\left(\mathrm{CDCl}_{3}, 300 \mathrm{MHz}\right)$ : $6.85(t, J=7.9 \mathrm{~Hz}, 1 \mathrm{H}), 7.79(d d, J=7.9$ and $1.6 \mathrm{~Hz}, 1 \mathrm{H}), 7.92$ $(d d, J=7.9$ and $1.6 \mathrm{~Hz}, 1 \mathrm{H}), 11.07(s, 1 \mathrm{H})$ p.p.m. ${ }^{13} \mathrm{C} \mathrm{NMR}$ $\left(\mathrm{CDCl}_{3}, 75 \mathrm{MHz}\right): 111.8,112.8,120.7(\mathrm{CH}), 130.6(\mathrm{CH}), 140.5$ (CH), 159.0, 174.1 p.p.m. IR (neat): 2855, 2526, 1653, 1603, $1428,1298,1243,1153,851,744,677,469 \mathrm{~cm}^{-1}$.

\section{Refinement}

Crystal data, data collection and structure refinement details are summarized in Table 3. Positions of hydrogen atoms bonded to carbon atoms were generated in idealized geometries using a riding model and their displacement parameters were set to $U_{\text {iso }}(\mathrm{H})=1.2 U_{\text {eq }}(\mathrm{C})$. The $\mathrm{H}$ atoms attached to $\mathrm{O}$ were identified from difference Fourier maps and their positions refined with restrained distances $[\mathrm{O}-\mathrm{H} 0.86$ (2) $\AA$ 品] and their isotropic thermal displacement parameters were refined freely.

\section{References}

Adam, M. S., Gutmann, M. J., Leech, C. K., Middlemiss, D. S., Parkin, A., Thomas, L. H. \& Wilson, C. C. (2010). New J. Chem. 34, 85-91. Agilent (2012). CrysAlis PRO. Agilent Technologies, Yarnton, England.

Bawa, S., Coté, M. L., Dubois, P., Lalancette, R. A. \& Thompson, H. W. (2004). Acta Cryst. B60, 438-446.

Bernstein, J., Davis, R. E., Shimoni, L. \& Chang, N.-L. (1995). Angew. Chem. Int. Ed. Engl. 34, 1555-1573.

Braun, D. E., Karamertzanis, P. G., Arlin, J.-B., Florence, A. J., Kahlenberg, V., Tocher, D. A., Griesser, U. J. \& Price, S. L. (2011). Cryst. Growth Des. 11, 210-220.

Choudhury, A. R. \& Guru Row, T. N. (2004). Acta Cryst. E60, o1595o1597.

Claude, R., Zarembowitch, J., Philoche-Levisalles, M. \& D'Yvoire, F. (1991). New J. Chem. 15, 635-641.

Cox, P. J. \& Murphy, M. T. (2003). Acta Cryst. E59, o1108-o1110.

Eijgendaal, I., Klein, G., Ter Horst-Van Amstel, M. J. L., Zwier, K., Bruins, N., Rigter, H. T. \& Gout, E. (2006). US Patent 0040932 A1.

Etter, M. C., MacDonald, J. C. \& Bernstein, J. (1990). Acta Cryst. B46, 256-262.

Farrugia, L. J. (2012). J. Appl. Cryst. 45, 849-854.

Gao, Z.-W., Zhang, C.-Y., Gao, L.-X., Wang, G.-F., Wu, D.-H. \& Liu, Z.-T. (2005). Z. Kristallogr. New Cryst. Struct. 220, 553-554.

Gelbrich, T. \& Hursthouse, M. B. (2005). CrystEngComm, 7, 324-336.

Gelbrich, T., Threlfall, T. L. \& Hursthouse, M. B. (2012). CrystEngComm, 14, 5454-5464.

Groom, C. R. \& Allen, F. H. (2014). Angew. Chem. Int. Ed. 53, 662671.

Laus, G., Kahlenberg, V., Wurst, K., Nerdinger, S. \& Schottenberger, H. (2011). Z. Naturforsch. Teil B, 66, 479-486.

Li, J.-H., Dong, F.-Y., Cai, F., Yuan, X.-F. \& Jiang, R.-W. (2012). Acta Cryst. E68, o825-o826.

Liu, C.-B., Chen, D.-D. \& Wen, H.-L. (2008). Acta Cryst. E64, o3.

Lu, Y.-B., Yang, P., Huang, W.-N., Yang, Y.-N. \& Wu, J.-Z. (2010). Acta Cryst. C66, o596-0599.

Macrae, C. F., Edgington, P. R., McCabe, P., Pidcock, E., Shields, G. P., Taylor, R., Towler, M. \& van de Streek, J. (2006). J. Appl. Cryst. 39, 453-457.

Meldrum, A. N. \& Shah, M. S. (1923). J. Chem. Soc. Trans. 123, 19861993.

Mereiter, K., Mikenda, W. \& Reichl, G. (2001). Private communication (refcode: IBUMAT). CCDC, Cambridge, England. 
Montis, R. \& Hursthouse, M. B. (2012). CrystEngComm, 14, 52425254.

Palatinus, L. \& Chapuis, G. (2007). J. Appl. Cryst. 40, 786-790.

Parkin, A., Adam, M., Cooper, R. I., Middlemiss, D. S. \& Wilson, C. C. (2007). Acta Cryst. B63, 303-308.

Sarma, B., Sanphui, P. \& Nangia, A. (2010). Cryst. Growth Des. 10, 2388-2399.
Sheldrick, G. M. (2008). Acta Cryst. A64, 112-122.

Sheldrick, G. M. (2015). Acta Cryst. C71, 3-8.

Talberg, H. J. (1977). Acta Chem. Scand. Ser. A, 31, 485-491.

Westrip, S. P. (2010). J. Appl. Cryst. 43, 920-925.

Zwier, K., Klein, G., Eijgendaal, I. \& Ter Horst-Van Amstel, M. J. L. (2005). Int. Patent WO 016898 A2. 


\section{supporting information}

Acta Cryst. (2015). E71, 531-535 [https://doi.org/10.1107/S2056989015007331]

\section{Crystal structure of 3-bromo-2-hydroxybenzoic acid}

\section{Gerhard Laus, Volker Kahlenberg, Thomas Gelbrich, Sven Nerdinger and Herwig Schottenberger}

Computing details

Data collection: CrysAlis PRO (Agilent, 2012); cell refinement: CrysAlis PRO (Agilent, 2012); data reduction: CrysAlis PRO (Agilent, 2012); program(s) used to solve structure: SUPERFLIP (Palatinus \& Chapuis, 2007); program(s) used to refine structure: SHELXL2014 (Sheldrick, 2015); molecular graphics: ORTEP-3 for Windows (Farrugia, 2012), Mercury (Macrae et al., 2006) and XP (Sheldrick, 2008); software used to prepare material for publication: WinGX (Farrugia, 2012) and publCIF (Westrip, 2010).

3-Bromo-2-hydroxybenzoic acid

Crystal data

$\mathrm{C}_{7} \mathrm{H}_{5} \mathrm{BrO}_{3}$

$M_{r}=217.02$

Monoclinic, $P 2_{1} / n$

$a=3.7978(4) \AA$

$b=10.5567(6) \AA$

$c=18.0366(10) \AA$

$\beta=90.208(7)^{\circ}$

$V=723.12(10) \AA^{3}$

$Z=4$

\section{Data collection}

Agilent Xcalibur (Ruby, Gemini ultra) diffractometer

Radiation source: Enhance (Mo) X-ray Source

Graphite monochromator

Detector resolution: 10.3575 pixels $\mathrm{mm}^{-1}$

$\omega$ scans

Absorption correction: multi-scan

(CrysAlis PRO; Agilent, 2012)

$T_{\min }=0.094, T_{\max }=1$

\section{Refinement}

Refinement on $F^{2}$

Least-squares matrix: full

$R\left[F^{2}>2 \sigma\left(F^{2}\right)\right]=0.040$

$w R\left(F^{2}\right)=0.100$

$S=1.08$

1594 reflections

108 parameters

2 restraints
$F(000)=424$

$D_{\mathrm{x}}=1.993 \mathrm{Mg} \mathrm{m}^{-3}$

Mo $K \alpha$ radiation, $\lambda=0.71073 \AA$

Cell parameters from 1701 reflections

$\theta=3.0-28.1^{\circ}$

$\mu=5.63 \mathrm{~mm}^{-1}$

$T=173 \mathrm{~K}$

Plate, colourless

$0.32 \times 0.16 \times 0.08 \mathrm{~mm}$

4627 measured reflections

1594 independent reflections

1309 reflections with $I>2 \sigma(I)$

$R_{\text {int }}=0.040$

$\theta_{\max }=29.1^{\circ}, \theta_{\min }=3.0^{\circ}$

$h=-4 \rightarrow 3$

$k=-12 \rightarrow 10$

$l=-21 \rightarrow 19$

Primary atom site location: iterative

Secondary atom site location: difference Fourier map

Hydrogen site location: inferred from neighbouring sites

$\mathrm{H}$ atoms treated by a mixture of independent and constrained refinement 
$w=1 /\left[\sigma^{2}\left(F_{\mathrm{o}}^{2}\right)+(0.0507 P)^{2}+0.2934 P\right]$

where $P=\left(F_{\mathrm{o}}^{2}+2 F_{\mathrm{c}}^{2}\right) / 3$

$(\Delta / \sigma)_{\max }<0.001$

$$
\Delta \rho_{\max }=1.19 \text { e } \AA^{-3}
$$

\section{Special details}

Geometry. All e.s.d.'s (except the e.s.d. in the dihedral angle between two 1.s. planes) are estimated using the full covariance matrix. The cell e.s.d.'s are taken into account individually in the estimation of e.s.d.'s in distances, angles and torsion angles; correlations between e.s.d.'s in cell parameters are only used when they are defined by crystal symmetry. An approximate (isotropic) treatment of cell e.s.d.'s is used for estimating e.s.d.'s involving l.s. planes.

Fractional atomic coordinates and isotropic or equivalent isotropic displacement parameters $\left(\hat{A}^{2}\right)$

\begin{tabular}{lllll}
\hline & $x$ & $y$ & $z$ & $U_{\text {iss }} / U_{\text {eq }}$ \\
\hline Br1 & $0.28846(10)$ & $0.31635(3)$ & $0.19518(2)$ & $0.02717(17)$ \\
O2 & $0.9783(9)$ & $0.1747(2)$ & $0.51138(16)$ & $0.0323(7)$ \\
H2 & $1.055(16)$ & $0.109(4)$ & $0.535(3)$ & $0.08(2)^{*}$ \\
O1 & $0.5137(8)$ & $0.1225(2)$ & $0.30358(15)$ & $0.0304(7)$ \\
H1 & $0.574(15)$ & $0.064(4)$ & $0.334(3)$ & $0.060(16)^{*}$ \\
O3 & $0.7802(8)$ & $0.0380(2)$ & $0.42729(15)$ & $0.0330(7)$ \\
C3 & $0.4674(10)$ & $0.3437(3)$ & $0.2917(2)$ & $0.0214(8)$ \\
C2 & $0.5652(10)$ & $0.2381(3)$ & $0.3340(2)$ & $0.0216(8)$ \\
C1 & $0.7081(10)$ & $0.2576(3)$ & $0.4042(2)$ & $0.0214(8)$ \\
C7 & $0.8233(10)$ & $0.1476(4)$ & $0.4484(2)$ & $0.0241(8)$ \\
C4 & $0.5058(10)$ & $0.4649(3)$ & $0.3191(2)$ & $0.0251(8)$ \\
H4 & 0.4369 & 0.5356 & 0.2899 & $0.030^{*}$ \\
C6 & $0.7467(10)$ & $0.3806(3)$ & $0.4318(2)$ & $0.0242(8)$ \\
H6 & 0.8432 & 0.3935 & 0.4799 & $0.029^{*}$ \\
C5 & $0.6454(11)$ & $0.4832(4)$ & $0.3896(2)$ & $0.0284(9)$ \\
H5 & 0.6711 & 0.5666 & 0.4087 & $0.034^{*}$ \\
& & & & \\
\hline
\end{tabular}

Atomic displacement parameters $\left(\AA^{2}\right)$

\begin{tabular}{lllllll}
\hline & $U^{11}$ & $U^{22}$ & $U^{33}$ & $U^{12}$ & $U^{13}$ & $U^{23}$ \\
\hline Br1 & $0.0292(3)$ & $0.0333(3)$ & $0.0190(3)$ & $0.00063(15)$ & $-0.00907(17)$ & $0.00363(14)$ \\
O2 & $0.049(2)$ & $0.0296(16)$ & $0.0187(16)$ & $0.0017(12)$ & $-0.0129(14)$ & $0.0054(11)$ \\
O1 & $0.0443(19)$ & $0.0232(15)$ & $0.0234(16)$ & $0.0006(12)$ & $-0.0131(13)$ & $0.0003(11)$ \\
O3 & $0.049(2)$ & $0.0252(15)$ & $0.0250(16)$ & $0.0053(12)$ & $-0.0134(13)$ & $0.0000(11)$ \\
C3 & $0.019(2)$ & $0.034(2)$ & $0.0118(19)$ & $0.0021(15)$ & $-0.0018(15)$ & $0.0026(14)$ \\
C2 & $0.0177(19)$ & $0.027(2)$ & $0.020(2)$ & $-0.0001(14)$ & $-0.0004(15)$ & $0.0025(14)$ \\
C1 & $0.0155(19)$ & $0.030(2)$ & $0.018(2)$ & $0.0012(14)$ & $0.0004(15)$ & $0.0031(14)$ \\
C7 & $0.022(2)$ & $0.035(2)$ & $0.015(2)$ & $0.0033(15)$ & $-0.0009(16)$ & $0.0018(15)$ \\
C4 & $0.023(2)$ & $0.027(2)$ & $0.026(2)$ & $0.0009(15)$ & $-0.0025(17)$ & $0.0022(15)$ \\
C6 & $0.026(2)$ & $0.028(2)$ & $0.018(2)$ & $-0.0012(15)$ & $-0.0051(16)$ & $-0.0004(14)$ \\
C5 & $0.033(2)$ & $0.020(2)$ & $0.031(2)$ & $-0.0008(15)$ & $-0.0031(18)$ & $-0.0001(14)$ \\
\hline
\end{tabular}

Geometric parameters $\left(A,{ }^{\circ}\right)$

\begin{tabular}{llll}
\hline $\mathrm{Br} 1-\mathrm{C} 3$ & $1.889(4)$ & $\mathrm{C} 2-\mathrm{C} 1$ & $1.391(5)$ \\
$\mathrm{O} 2-\mathrm{C} 7$ & $1.309(5)$ & $\mathrm{C} 1-\mathrm{C} 6$ & $1.398(6)$
\end{tabular}




\begin{tabular}{|c|c|c|c|}
\hline $\mathrm{O} 2-\mathrm{H} 2$ & $0.87(2)$ & $\mathrm{C} 1-\mathrm{C} 7$ & $1.474(5)$ \\
\hline $\mathrm{O} 1-\mathrm{C} 2$ & $1.352(5)$ & $\mathrm{C} 4-\mathrm{C} 5$ & $1.388(6)$ \\
\hline $\mathrm{O} 1-\mathrm{H} 1$ & $0.85(2)$ & $\mathrm{C} 4-\mathrm{H} 4$ & 0.9500 \\
\hline $\mathrm{O} 3-\mathrm{C} 7$ & $1.229(5)$ & $\mathrm{C} 6-\mathrm{C} 5$ & $1.379(5)$ \\
\hline $\mathrm{C} 3-\mathrm{C} 4$ & $1.379(5)$ & $\mathrm{C} 6-\mathrm{H} 6$ & 0.9500 \\
\hline $\mathrm{C} 3-\mathrm{C} 2$ & $1.400(5)$ & $\mathrm{C} 5-\mathrm{H} 5$ & 0.9500 \\
\hline $\mathrm{C} 7-\mathrm{O} 2-\mathrm{H} 2$ & $114(4)$ & $\mathrm{O} 3-\mathrm{C} 7-\mathrm{C} 1$ & $122.4(3)$ \\
\hline $\mathrm{C} 2-\mathrm{O} 1-\mathrm{H} 1$ & $111(4)$ & $\mathrm{O} 2-\mathrm{C} 7-\mathrm{C} 1$ & $115.4(3)$ \\
\hline $\mathrm{C} 4-\mathrm{C} 3-\mathrm{C} 2$ & $121.0(4)$ & $\mathrm{C} 3-\mathrm{C} 4-\mathrm{C} 5$ & $119.8(3)$ \\
\hline $\mathrm{C} 4-\mathrm{C} 3-\mathrm{Br} 1$ & $120.7(3)$ & $\mathrm{C} 3-\mathrm{C} 4-\mathrm{H} 4$ & 120.1 \\
\hline $\mathrm{C} 2-\mathrm{C} 3-\mathrm{Br} 1$ & $118.3(3)$ & $\mathrm{C} 5-\mathrm{C} 4-\mathrm{H} 4$ & 120.1 \\
\hline $\mathrm{O} 1-\mathrm{C} 2-\mathrm{C} 1$ & $123.9(3)$ & $\mathrm{C} 5-\mathrm{C} 6-\mathrm{C} 1$ & $120.3(4)$ \\
\hline $\mathrm{O} 1-\mathrm{C} 2-\mathrm{C} 3$ & $117.4(3)$ & $\mathrm{C} 5-\mathrm{C} 6-\mathrm{H} 6$ & 119.9 \\
\hline $\mathrm{C} 1-\mathrm{C} 2-\mathrm{C} 3$ & $118.7(3)$ & $\mathrm{C} 1-\mathrm{C} 6-\mathrm{H} 6$ & 119.9 \\
\hline $\mathrm{C} 2-\mathrm{C} 1-\mathrm{C} 6$ & $120.1(3)$ & $\mathrm{C} 6-\mathrm{C} 5-\mathrm{C} 4$ & $120.1(3)$ \\
\hline $\mathrm{C} 2-\mathrm{C} 1-\mathrm{C} 7$ & $119.3(3)$ & $\mathrm{C} 6-\mathrm{C} 5-\mathrm{H} 5$ & 120.0 \\
\hline $\mathrm{C} 6-\mathrm{C} 1-\mathrm{C} 7$ & $120.6(3)$ & $\mathrm{C} 4-\mathrm{C} 5-\mathrm{H} 5$ & 120.0 \\
\hline $\mathrm{O} 3-\mathrm{C} 7-\mathrm{O} 2$ & $122.2(3)$ & & \\
\hline $\mathrm{C} 4-\mathrm{C} 3-\mathrm{C} 2-\mathrm{O} 1$ & $178.9(4)$ & $\mathrm{C} 6-\mathrm{C} 1-\mathrm{C} 7-\mathrm{O} 3$ & $176.9(4)$ \\
\hline $\mathrm{Br} 1-\mathrm{C} 3-\mathrm{C} 2-\mathrm{O} 1$ & $-2.2(5)$ & $\mathrm{C} 2-\mathrm{C} 1-\mathrm{C} 7-\mathrm{O} 2$ & $175.1(3)$ \\
\hline $\mathrm{C} 4-\mathrm{C} 3-\mathrm{C} 2-\mathrm{C} 1$ & $-1.0(6)$ & $\mathrm{C} 6-\mathrm{C} 1-\mathrm{C} 7-\mathrm{O} 2$ & $-3.8(5)$ \\
\hline $\mathrm{Br} 1-\mathrm{C} 3-\mathrm{C} 2-\mathrm{C} 1$ & $177.9(3)$ & $\mathrm{C} 2-\mathrm{C} 3-\mathrm{C} 4-\mathrm{C} 5$ & $0.4(6)$ \\
\hline $\mathrm{O} 1-\mathrm{C} 2-\mathrm{C} 1-\mathrm{C} 6$ & $-178.9(3)$ & $\mathrm{Br} 1-\mathrm{C} 3-\mathrm{C} 4-\mathrm{C} 5$ & $-178.5(3)$ \\
\hline $\mathrm{C} 3-\mathrm{C} 2-\mathrm{C} 1-\mathrm{C} 6$ & $1.0(5)$ & $\mathrm{C} 2-\mathrm{C} 1-\mathrm{C} 6-\mathrm{C} 5$ & $-0.3(6)$ \\
\hline $\mathrm{O} 1-\mathrm{C} 2-\mathrm{C} 1-\mathrm{C} 7$ & $2.1(6)$ & $\mathrm{C} 7-\mathrm{C} 1-\mathrm{C} 6-\mathrm{C} 5$ & $178.6(4)$ \\
\hline $\mathrm{C} 3-\mathrm{C} 2-\mathrm{C} 1-\mathrm{C} 7$ & $-178.0(3)$ & $\mathrm{C} 1-\mathrm{C} 6-\mathrm{C} 5-\mathrm{C} 4$ & $-0.3(6)$ \\
\hline $\mathrm{C} 2-\mathrm{C} 1-\mathrm{C} 7-\mathrm{O} 3$ & $-4.2(6)$ & $\mathrm{C} 3-\mathrm{C} 4-\mathrm{C} 5-\mathrm{C} 6$ & $0.3(6)$ \\
\hline
\end{tabular}

Hydrogen-bond geometry $\left(A,{ }^{o}\right)$

\begin{tabular}{lllll}
\hline$D-\mathrm{H} \cdots A$ & $D-\mathrm{H}$ & $\mathrm{H} \cdots A$ & $D \cdots A$ & $D-\mathrm{H} \cdots A$ \\
\hline $\mathrm{O} 1-\mathrm{H} 1 \cdots \mathrm{O} 3$ & $0.85(2)$ & $1.88(4)$ & $2.604(4)$ & $142(5)$ \\
$\mathrm{O} 2-\mathrm{H} 2 \cdots \mathrm{O} 3{ }^{\mathrm{i}}$ & $0.87(2)$ & $1.80(2)$ & $2.664(4)$ & $172(6)$ \\
\hline
\end{tabular}

Symmetry code: (i) $-x+2,-y,-z+1$. 